\title{
Disparition rapide de la grippe après la mise en œuvre de mesures d'atténuation de la COVID-19 à Hamilton, Ontario
}

\author{
Kevin Zhang ${ }^{1 \star}$, Avika Misra², Patrick J Kim³ ${ }^{3}$ Seyed M Moghadas ${ }^{4}$, Joanne M Langley5, \\ Marek Smieja ${ }^{3,6}$
}

\section{Résumé}

Contexte : Des mesures de santé publique, telles que la distanciation physique et la fermeture des écoles et des services non essentiels, ont été rapidement mises en œuvre au Canada pour freiner la propagation de la maladie à coronavirus 2019 (COVID-19). Nous avons cherché à étudier les conséquences des mesures d'atténuation pendant la vague printanière de COVID-19 sur l'incidence d'autres virus respiratoires confirmés en laboratoire à Hamilton, en Ontario.

Méthodes : Tous les échantillons d'écouvillons nasopharyngés ( $n=57503$ ) soumis à un dépistage systématique du virus respiratoire dans un laboratoire régional desservant tous les centres hospitaliers de soins de courte durée de Hamilton entre janvier 2010 et juin 2020 ont été examinés. Les tests de dépistage de la grippe $A$ et $B$, du virus respiratoire syncytial, du métapneumovirus humain, du virus para-influenza I-III, de l'adénovirus et du rhinovirus/ entérovirus ont été effectués systématiquement à l'aide d'un panel multiplex de virus respiratoires par réaction en chaîne par polymérase mis au point par le laboratoire. Un modèle de régression linéaire bayésien a été utilisé pour déterminer la tendance des taux de positivité de tous les échantillons de grippe au cours des 26 premières semaines de chaque année de 2010 à 2019. Le taux de positivité moyen de l'inférence bayésienne a été comparé au taux de positivité hebdomadaire des échantillons de grippe rapporté en 2020.

Résultats : Le taux de positivité de la grippe en 2020 a fortement diminué après la mise en œuvre d'interventions relatives à la COVID-19 à l'échelle de la population. Les semaines 12-26 ont rapporté $0 \%$ de positivité pour la grippe, à l'exception de 0,1\% rapporté la semaine 13 .
Cette oeuvre est mise à la disposition selon les termes de la licence internationale Creative Commons Attribution 4.0

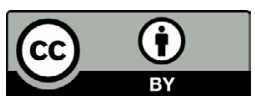

Affiliations

${ }^{1}$ Faculté de médecine, Université de Toronto, Toronto, ON

${ }^{2}$ Faculté de médecine, Université d'Ottawa, Ottawa, ON

${ }^{3}$ Département de médecine, Université McMaster, Hamilton, ON

${ }^{4}$ Agent-Based Modelling Laboratory, York University, Toronto, ON

${ }^{5}$ Canadian Center for Vaccinology, Université Dalhousie, IWK Health Centre et Nova Scotia Health Authority, Halifax, NS

${ }^{6}$ St. Joseph's Healthcare Hamilton, Hamilton, ON

\section{*Correspondance :}

kevink.zhang@mail.utoronto.ca

Conclusion : Les mesures de santé publique mises en œuvre pendant la pandémie de COVID-19 ont été associées à une incidence réduite d'autres virus respiratoires et devraient être envisagées pour atténuer la gravité de la grippe saisonnière et des pandémies dues à d'autres virus respiratoires.

Citation proposée : Zhang K, Misra A, Kim PJ, Moghadas SM, Langley JM, Smieja M. Disparition rapide de la grippe après la mise en œuvre de mesures d'atténuation de la COVID-19 à Hamilton, Ontario. Relevé des maladies transmissibles au Canada 2021;47(4):221-7. https://doi.org/10.14745/ccdr.v47i04a04f

Mots-clés : COVID-19, grippe, dépistage, interventions de santé publique, Canada

\section{Introduction}

La maladie à coronavirus 2019 (COVID-19) a entraîné une morbidité et une mortalité mondiales dévastatrices (1). Des mesures de santé publique restrictives ont permis d'atténuer la transmission de la COVID-19 (2,3), mais ont entraîné des perturbations généralisées de l'économie $(4,5)$, du commerce (6) et de l'éducation (7). Lorsque l'Organisation mondiale de la Santé a déclaré que la COVID-19 était une pandémie le
11 mars 2020 (8), la province de l'Ontario, au Canada, a annoncé la fermeture de toutes les écoles et des lieux de travail non essentiels $(9,10)$. Des mois plus tard, des mesures de santé publique, telles que la distanciation physique et le port de masques, continuent d'être mises en place pour réduire le bilan associé à la pandémie de COVID-19 (11). 
Les mesures de santé publique ont permis de réduire la transmission du coronavirus du syndrome respiratoire aigu sévère 2 (SRAS-CoV-2) en Ontario (3). Dans certaines régions, ces mesures ont également été associées à une incidence plus faible d'autres infections par des virus respiratoires $(12,13)$. Nous avons effectué une analyse par séries chronologiques, à l'aide d'un modèle de régression hiérarchique, pour déterminer les calendriers et les taux de positivité des virus de la grippe A et $\mathrm{B}$ de 2010 à 2019 dans un centre urbain de l'Ontario, et les comparer à ceux de 2020 avant et après la mise en œuvre des interventions relatives à la COVID-19 en réponse aux éclosions initiales.

\section{Méthodes}

\section{Échantillonnage et tests}

Nous avons examiné tous les échantillons d'écouvillons nasopharyngés ( $n=57$ 503) soumis à un dépistage systématique des virus respiratoires dans un laboratoire régional desservant tous les centres hospitaliers de soins de courte durée Hamilton, en Ontario, entre janvier 2010 et juin 2020.

Les tests ont été effectués à l'aide d'un panel multiplex de virus respiratoires par $\mathrm{PCR}$ après transcription inverse en temps réel grâce à la technologie TaqMan, mise au point par le programme régional de médecine de laboratoire de Hamilton, pour la grippe $A$ et $B$, le virus respiratoire syncytial, le métapneumovirus humain, le virus para-influenza I-III, I'adénovirus et le rhinovirus/ entérovirus. Le 16 mars 2020, le virus para-influenza II a été remplacé par le virus SRAS-CoV-2. L'extraction et l'amplification de l'acide ribonucléique (ARN) des échantillons ont été principalement réalisées sur les appareils bioMérieux NucliSENS easyMag et QIAGEN Rotor-Gene Q, respectivement, de 2010 à 2019 et principalement sur le système BD MAX de juillet 2019 à 2020. Les résultats cliniques ont été validés par un personnel expérimenté et consignés dans un système d'information de laboratoire, conformément aux procédures opérationnelles normalisées.

\section{Données}

Une base de données sur les virus respiratoires contenant tous les résultats des tests et des informations démographiques est mise à jour chaque semaine et est en place depuis 2010. Une saisie des données sur 10 ans avec des informations démographiques de base (âge, sexe, code postal, date, établissement, numéro d'enregistrement) et des résultats de tests a été exportée de la base de données du laboratoire le 29 juin 2020. La base de données ne comprenait que les échantillons envoyés pour le test multiplex. Les résultats des tests de laboratoire ont été filtrés par code postal afin d'exclure les échantillons provenant de personnes vivant en dehors de Hamilton.

\section{Approbation éthique}

L'étude a été approuvée par le comité d'éthique de la recherche intégré de Hamilton (projet : 07-2923). L'étude a été classée comme étant à risque minimal, c'est-à-dire sans risque de conséquence négative sur la santé et la sécurité du participant, et une renonciation au consentement individuel de participation a été obtenue.

\section{Analyse statistique}

Nous avons utilisé un modèle de régression linéaire bayésien avec des distributions préalables non informatives pour déterminer la tendance des taux de positivité de tous les échantillons de grippe $A$ et $B$ au cours des 26 premières semaines de chaque année de 2010 à 2019 (annexe :

tableau A1). Nous avons ensuite comparé le taux de positivité moyen de l'inférence bayésienne avec le taux de positivité hebdomadaire des échantillons de grippe rapporté en 2020 (annexe : tableau A2).

Le modèle de régression hiérarchique a la forme suivante

$$
\begin{aligned}
& y \sim \operatorname{Normal}(\mu, \sigma) \\
& \mu=\beta^{\top} \boldsymbol{x} \\
& \beta=\operatorname{Normal}(0,100) \\
& \sigma^{2}=\operatorname{InverseGamma}(2.5,25)
\end{aligned}
$$

où y représente le taux de positivité au cours des 26 premières semaines (variable $x$ ) de chaque année de 2010 à 2019. Tous les paramètres ont été échantillonnés à l'aide de simulations MCMC (Markov Chain Monte Carlo) dans trois chaînes indépendantes. Chaque chaîne comprenait 10000 itérations, avec une période de rodage de 1000 itérations et un facteur de dilution de cinq. Pour évaluer la convergence, nous avons inspecté les tracés et appliqué le test de convergence de Gelman-Rubin en calculant les facteurs potentiels de réduction d'échelle (FPRE). Toutes les valeurs des FPRE ont été calculées pour être inférieures à 1,1 (et sont restées proches de 1), indiquant la convergence des paramètres du modèle vers leurs distributions postérieures. Nous avons utilisé les distributions postérieures des paramètres $(\beta 1, \beta 2, \sigma)$ de notre analyse bayésienne pour dériver des estimations moyennes et des intervalles de crédibilité (annexe : tableau A3) en employant la méthode de la plus haute densité postérieure (14).

\section{Résultats}

Une description des individus compris dans notre étude est fournie dans le tableau 1. Au total, 48459 patients ont été testés pour des virus respiratoires à Hamilton, en Ontario, entre 2010 et 2019 , dont $49,3 \%(n=23898)$ étaient des hommes et 30,6 $\%(n=14818)$ des enfants de moins de 18 ans. La distribution bimodale des âges présentait un âge médian des adultes de $72,4$ ans ( $E I: 59,4-83,5)$ et 1,5 an chez les enfants ( $E I: 0,4-4,4)$. 
Une médiane de 4626 (IQR : 3 376-5 936) échantillons ont été testés chaque année, avec un taux de positivité moyen pour la grippe de 9,6\% (écart-type : $2.9 \%$ ). Le pourcentage moyen de positivité a également été calculé pour le virus respiratoire syncytial (6,9\%, écart-type : 1,5\%), le métapneumovirus $(2,8 \%$, écart-type : $0,4 \%)$, le virus para-influenza $(3,2 \%$, écart-type : $0,6 \%$ ), adénovirus (1,0\%, écart-type : $0,6 \%$ ), et les rhinovirus/ entérovirus (8,0\%, écart-type : 5,5\%). Au total, 9044 patients ont été testés pour des virus respiratoires en 2020 , dont $2,5 \%$ étaient positifs pour la grippe. Le pourcentage de positivité des autres virus respiratoires variait de 0,1\% (virus para-influenza) à $0,9 \%$ (virus respiratoire syncytial et rhinovirus/entérovirus).

Tableau 1 : Données démographiques, taille de l'échantillon et taux de positivité des virus respiratoires confirmés en laboratoire à Hamilton (Ontario) en 2010-2019 ( $n=48459)$ et 2020 ( $n=9$ 044)

\begin{tabular}{|c|c|c|c|c|}
\hline \multirow{2}{*}{$\begin{array}{l}\text { Description } \\
\text { Âge en années }\end{array}$} & \multicolumn{2}{|c|}{2010 à 2019} & \multicolumn{2}{|c|}{2020} \\
\hline & Médiane & El & Médiane & EI \\
\hline Adultes & 72,4 & $59,4-83,5$ & 63,0 & $46,1-77,2$ \\
\hline Enfants & 1,5 & $0,4-4,4$ & 1,9 & $0,5-6,0$ \\
\hline $\begin{array}{c}\text { Données } \\
\text { démographiques }\end{array}$ & Nombre & $\%$ & Nombre & $\%$ \\
\hline Homme & 23898 & $49,3 \%$ & 4073 & $45,0 \%$ \\
\hline Adultes & 33641 & $69,4 \%$ & 7983 & $88,3 \%$ \\
\hline Enfants & 14818 & $30,6 \%$ & 1061 & $11,7 \%$ \\
\hline $\begin{array}{l}\text { Échantillons } \\
\text { de virus } \\
\text { respiratoires }\end{array}$ & Médiane & El & Nor & bre \\
\hline $\begin{array}{l}\text { Échantillons par } \\
\text { an }\end{array}$ & 4626 & $3376-5936$ & & 9044 \\
\hline $\begin{array}{l}\text { Taux de } \\
\text { positivité }\end{array}$ & Moyenne & Écart-type & Moy & nne \\
\hline Grippe & $9,6 \%$ & $2,9 \%$ & & $2,5 \%$ \\
\hline $\begin{array}{l}\text { Virus respiratoire } \\
\text { syncytial }\end{array}$ & $6,9 \%$ & $1,5 \%$ & & $0,9 \%$ \\
\hline Métapneumovirus & $2,8 \%$ & $0,4 \%$ & & $0,4 \%$ \\
\hline $\begin{array}{l}\text { Virus para- } \\
\text { influenza }\end{array}$ & $3,2 \%$ & $0,6 \%$ & & $0,1 \%$ \\
\hline Adénovirus & $1,0 \%$ & $0,6 \%$ & & $0,2 \%$ \\
\hline $\begin{array}{l}\text { Rhinovirus/ } \\
\text { entérovirus }\end{array}$ & $8,0 \%$ & $5,5 \%$ & & $0,9 \%$ \\
\hline
\end{tabular}

La figure 1 montre les taux de positivité de la grippe $A$ et $B$ dans la base de données pour les différents groupes d'âge.

La figure 2 illustre le taux de positivité moyen dérivé des distributions postérieures des paramètres du modèle de régression linéaire bayésien en utilisant les taux de positivité rapportés de 2010 à 2019 (courbe noire). Le taux de positivité de la grippe en 2020 (courbe rouge) a été le plus élevé (17,7\%)
Figure 1 : Taux de positivité de la grippe $A$ et $B$ de 2010 à 2020 pour différents groupes d'âge à Hamilton, Ontario

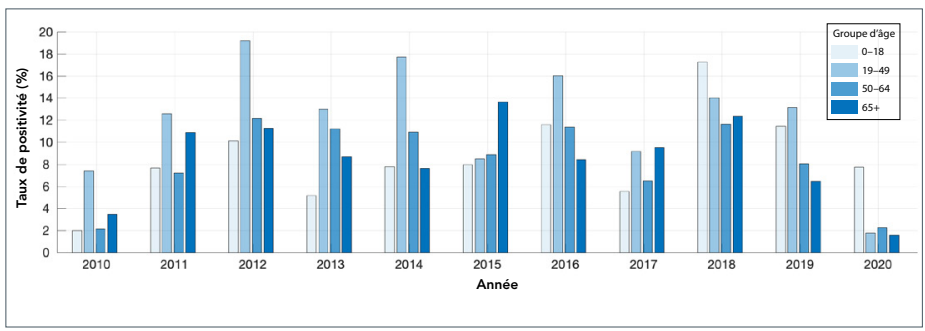

au cours de la première semaine, et a chuté en dessous de l'intervalle de crédibilité à $95 \%$ pour les 10 années précédentes après la première semaine, avec une tendance à la baisse par la suite (figure 2; annexe : tableau A2). Après la mise en œuvre des interventions relatives à la COVID-19 au cours de la semaine 12 (à partir du 12 mars 2020; barre grise dans la figure 2), le taux de positivité de la grippe a fortement diminué et est resté à $0 \%$ au cours des semaines 12 à 26 , à l'exception de $0,1 \%$ signalé au cours de la semaine 13 .

Figure 2 : Inférence bayésienne pour le taux de positivité moyen et son intervalle de crédibilité à $95 \%$ de la grippe $A$ et $B$ au cours des 26 premières semaines de 2010 à 2019

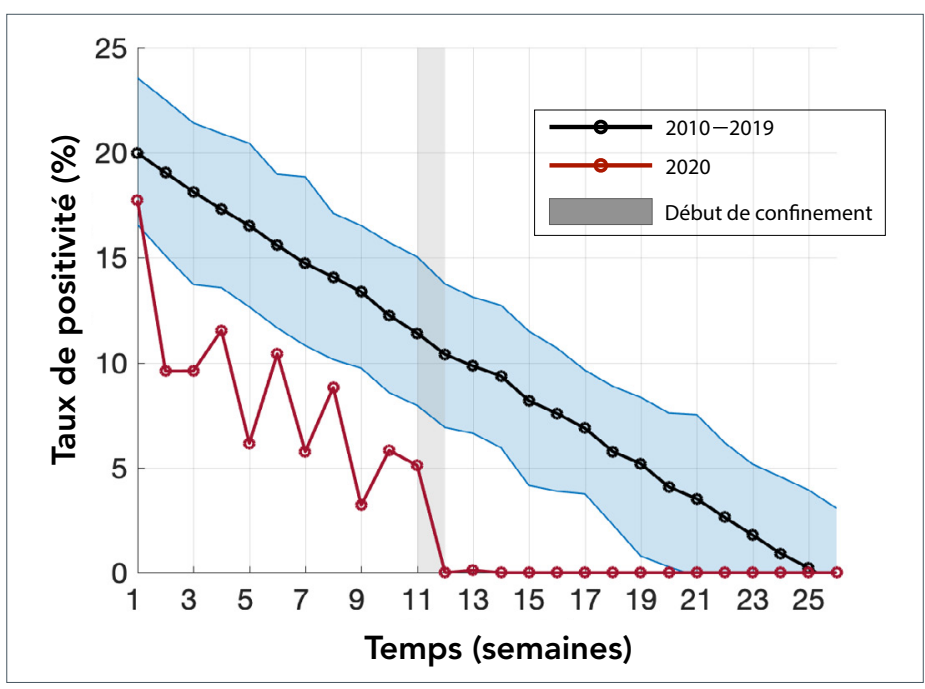

Remarque : La courbe rouge montre le taux de positivité de la grippe A et B pour 2020, la barre grise ombrée indiquant le début du confinement dû à la COVID-19

\section{Discussion}

Des mesures de santé publique ont été mises en place pour freiner la propagation de la grippe pendant les pandémies, avec des niveaux de réussite variables. Par exemple, la fermeture des écoles et la distanciation physique pendant la pandémie de grippe H1N1 de 2009 au Mexique ont permis de réduire de $27 \%$ à $29 \%$ la transmission de la grippe pendant la vague du 
printemps (15). Pendant la pandémie de grippe de 1957 à 1958 la fermeture des écoles a contribué à réduire le taux d'attaque de plus de 90 \% (16). De même, après la mise en œuvre de mesures d'atténuation du taux de positivité COVID-19, le taux de positivité de la grippe a été supprimé aux États-Unis $(12,13)$. Nos résultats suggèrent que les mesures de santé publique concernant la COVID-19 ont pu contribuer à une perturbation substantielle de la propagation de la grippe à Hamilton.

La saison grippale 2020 a été observée comme étant relativement douce à Hamilton, par rapport aux saisons précédentes (annexe : tableau A1, A2). Cependant, le taux de positivité plus faible observé dans notre analyse (figure 2), peut être attribué à plusieurs facteurs, notamment les précautions volontaires prises par les individus à la suite des premiers reportages sur la propagation de la COVID-19 en Chine et dans le monde, les variations saisonnières normales, ou les changements dans le comportement d'échantillonnage et les tests de diagnostic. Pour la saison hivernale de la grippe de 2010 à 2019, le taux médian de positivité de la grippe a atteint $0 \%$ à la semaine 23. En 2020, cependant, après la mise en œuvre des mesures d'atténuation de la COVID-19, le pourcentage de positivité pour la grippe a chuté abruptement pour atteindre $0 \%$ à la semaine 12. Les Centers for Disease Control and Prevention $(C D C)$ ont fait état de résultats similaires dans le cadre de leur système de surveillance hebdomadaire de la grippe, dans lequel le pourcentage de positivité pour la grippe est passé de 7,5\% à la semaine 12 à 1,0\% à la semaine 14. Ce changement brusque, sans autre explication, suggère que les mesures d'atténuation de la COVID-19 pourraient avoir réduit la propagation de la grippe confirmée en laboratoire aux États-Unis $(12,13)$. De plus, les taux de positivité pour le virus respiratoire syncytial, le métapneumovirus, le virus para-influenza, l'adénovirus et le rhinovirus/entérovirus ont été rapportés comme étant de $0 \%$ à la semaine 14 de 2020 (annexe : tableau A2), ce qui suggère que les mesures de santé publique auraient également pu supprimer la transmission d'autres virus respiratoires.

La compréhension de l'effet des interventions concernant la COVID-19 sur d'autres maladies transmissibles nécessite une étude plus approfondie. Un certain nombre de facteurs peuvent être envisagés pour décrire l'interruption rapide des chaînes de transmission de la grippe par rapport à COVID-19 en raison de la pression exercée par les mesures de santé publique. Premièrement, il existe une immunité croisée relativement forte pour les souches du virus de la grippe pendant les épidémies saisonnières, en plus de l'immunité de la population conférée par la vaccination $(17,18)$. En revanche, la population était naïve face au SRAS-CoV-2 et reste largement sensible en l'absence de vaccination. En outre, il existe des différences majeures dans les caractéristiques épidémiologiques entre la grippe et la COVID-19 qui influencent les résultats des interventions (19). Par exemple, on estime que la transmissibilité de la grippe se situe entre 1,2 et 1,8 (20), ce qui est inférieur aux estimations initiales de plus de deux pour la COVID-19 dans la plupart des contextes $(21,22)$. La période d'incubation moyenne de 5,2 jours pour la COVID-19 (21) est nettement plus longue que la même période pour la grippe, estimée à 1,4 jour (23). De plus, la période présymptomatique est plus longue et plus infectieuse dans le cas de la COVID-19 que dans celui de la grippe $(24,25)$. Les études futures devront tenir compte de ces facteurs lors de l'évaluation de l'effet des interventions contre les maladies infectieuses émergentes.

Les résultats de notre étude doivent être interprétés dans le contexte des limites de l'étude. Tout d'abord, les échantillons respiratoires n'ont pas été recueillis de manière systématique, mais plutôt dans le cadre de soins cliniques de routine. Il est donc possible que les échantillons ne soient pas totalement représentatifs de la prévalence des virus respiratoires dans la région. Il est également possible que les cliniciens n'aient pas suivi strictement la politique de contrôle des infections de l'hôpital et n'aient pas échantillonné des patients qui auraient autrement été éligibles. De plus, le comportement d'échantillonnage peut avoir changé pendant la phase initiale de la propagation de la COVID-19 au Canada. Cependant, il est peu probable que ces facteurs modifient nos conclusions en raison de la quasi-élimination du nombre absolu de cas de virus respiratoires confirmés en laboratoire, malgré la forte augmentation des tests qui a accompagné l'inquiétude suscitée par la COVID-19 dans la communauté.

\section{Conclusion}

Nos résultats suggèrent que les efforts pour contrôler la pandémie de COVID-19 ont pu avoir des avantages supplémentaires en supprimant la transmission d'autres virus respiratoires à Hamilton, en Ontario. Les stratégies d'atténuation, telles que la distanciation physique, le port du masque et la fermeture des écoles, pourraient jouer un rôle important dans la lutte contre les futurs virus respiratoires saisonniers et les maladies infectieuses émergentes à potentiel pandémique.

\section{Déclaration des auteurs}

K. Z., A. M., P. J. K., S. M. M. et M. S. ont contribué à la conception et à la réalisation de ce travail

K. Z., S. M. M., J. M. L. et M. S. ont contribué à l'acquisition des données, à l'analyse et à l'interprétation des résultats

Tous les auteurs ont rédigé, lu et approuvé le manuscrit final.

Le contenu de l'article et les points de vue qui y sont exprimés n'engagent que les auteurs et ne correspondent pas nécessairement à ceux du gouvernement du Canada.

\section{Intérêts concurrents}

La Dre J. Langley signale que I'Université Dalhousie a reçu des paiements de Sanofi, Glaxo-SmithKline, Merck, Janssen, VBI et Pfizer pour la réalisation d'études sur les vaccins. La Dre Langley est titulaire de la chaire des Instituts de recherche en santé du Canada et GlaxoSmithKline en vaccinologie pédiatrique. Aucun autre intérêt concurrent n'a été déclaré. 


\section{Financement}

S. Moghadas : IRSC (OV4 - 170643), Recherche pour I'intervention rapide contre la COVID-19; Conseil de recherches en sciences naturelles et en génie du Canada; et Fondation canadienne pour l'innovation.

\section{Références}

1. Johns Hopkins University of Medicine. Coronavirus Resource Center. COVID-19 Dashboard by the Center for Systems Science and Engineering (CSSE) (accédé 2020-09-01). https://coronavirus.jhu. edu/map.html

2. Zhang K, Vilches TN, Tariq M, Galvani AP, Moghadas SM. The impact of mask-wearing and shelter-in-place on COVID-19 outbreaks in the United States. Int J Infect Dis 2020;101:334-41. DOI PubMed

3. Santé publique Ontario. Séroprévalence de la COVID-19 en Ontario : du 27 mars 2020 au 30 juin 2020. SPO; 2020. https://www. publichealthontario.ca/-/media/documents/ncov/epi/2020/07/covid19-epi-seroprevalence-in-ontario.pdf?la=FR

4. Fernandes N. Economic Effects of Coronavirus Outbreak (COVID-19) on the World Economy. IESE Business School. 2020 March 22; Working Paper No. WP-1240-E. DOI

5. Jones L, Palumbo D, Brown D. Coronavirus: A visual guide to the economic impact. BBC News. 2020 Jun 30 (accédé 2020-08-17). https://www.bbc.com/news/business-51706225

6. Organisation mondiale du commerce. La COVID-19 et le commerce mondial. Genève (Suisse): OMS; 2020 (accédé 2020-08-17). https:// www.wto.org/french/tratop_f/covid19_f/covid19_f.htm

7. Dorn E, Hancock B, Sarakatsannis J, Viruleg E. New evidence shows that the shutdowns caused by COVID-19 could exacerbate existing achievement gaps. McKinsey and Company; 2020 June 1 (accédé 2020-08-17). https://www.mckinsey.com/industries/ public-and-social-sector/our-insights/covid-19-and-student-learnin $\mathrm{g}$-in-the-united-states-the-hurt-could-last-a-lifetime\#

8. Organisation monidale de la Santé. Allocution liminaire du Directeur général de l'OMS lors du point presse sur la COVID-19 - 11 mars 2020. Genève (Suisse) : OMS; mars 2020 (accédé 2020-08-17). https://www.who.int/fr/director-general/speeches/detail/ who-director-general-s-opening-remarks-at-the-media-briefi ng-on-covid-19---11-march-2020

9. Rodrigues G. Ontario government declares state of emergency amid coronavirus pandemic. Global News. 2020 March 17 (accédé 2020-08-17). https://globalnews.ca/news/6688074/ ontario-doug-ford-coronavirus-covid-19-march-17/

10. Vogel L. COVID-19: A timeline of Canada's first-wave response. CMAJ News. 2020 June 17 (accédé 2020-08-17). https://cmajnews. com/2020/06/12/coronavirus-1095847/

11. Gouvernement de l'Ontario. Déconfinement de l'Ontario (accédé 2020-08-17). https://www.ontario.ca/fr/page/deconfinement-delontario
12. Centers for Disease Control and Prevention. Influenza Positive Tests Reported to CDC by U.S. Clinical Laboratories, 2019-2020 Season. CDC; 2020 (accédé 2020-08-17). https://www.cdc.gov/flu/weekly/ weeklyarchives2019-2020/data/whoAllregt_cl32.html

13. Blum K. Was Social Distancing a Help in Slowing the Flu Season? Infectious Disease Special Edition. April 3, 2020 (accédé 2020-08-17). https://www.idse.net/Covid-19/Article/04-20/ Was-Social-Distancing-a-Help-in-Slowing-the-Flu-Season/57839?ses=ogst

14. Chen MH, Shao QM. Monte Carlo Estimation of Bayesian Credible and HPD Intervals. J Comput Graph Stat 1999;8(1):69-92. DOI

15. Stephenson J. Social distancing helpful in Mexico during flu pandemic. JAMA 2011;305(24):2509. DOI PubMed

16. Glass RJ, Glass LM, Beyeler WE, Min HJ. Targeted social distancing design for pandemic influenza. Emerg Infect Dis 2006;12(11):1671-81. DOI PubMed

17. Valkenburg SA, Rutigliano JA, Ellebedy AH, Doherty PC, Thomas PG, Kedzierska K. Immunity to seasonal and pandemic influenza A viruses. Microb Infect. 2011;13(5):489-501. https://linkinghub.elsevier.com/retrieve/pii/S1286457911000372

18. Krammer $F$. The human antibody response to influenza $A$ virus infection and vaccination. Nat Rev Immunol 2019;19(6):383-97. DOl PubMed

19. Abdollahi E, Haworth-Brockman M, Keynan Y, Langley JM, Moghadas SM. Simulating the effect of school closure during COVID-19 outbreaks in Ontario, Canada. BMC Med 2020;18(1):230. DOI PubMed

20. 20. Biggerstaff M, Cauchemez S, Reed C, Gambhir M, Finelli L. Estimates of the reproduction number for seasonal, pandemic, and zoonotic influenza: a systematic review of the literature. BMC Infect Dis 2014;14:480. DOI PubMed

21. Li Q, Guan $X$, Wu $P$, Wang $X$, Zhou $L$, Tong $Y$, Ren $R$, Leung KS, Lau EH, Wong JY, Xing X, Xiang N, Wu Y, Li C, Chen Q, Li D, Liu T, Zhao J, Liu M, Tu W, Chen C, Jin L, Yang R, Wang Q, Zhou S, Wang R, Liu H, Luo Y, Liu Y, Shao G, Li H, Tao Z, Yang Y, Deng Z, Liu B, Ma Z, Zhang Y, Shi G, Lam TT, Wu JT, Gao GF, Cowling BJ, Yang B, Leung GM, Feng Z. Early Transmission Dynamics in Wuhan, China, of Novel Coronavirus-Infected Pneumonia. N Engl J Med 2020;382(13):1199-207. DOI PubMed

22. Wu JT, Leung K, Leung GM. Nowcasting and forecasting the potential domestic and international spread of the 2019-nCoV outbreak originating in Wuhan, China: a modelling study. Lancet 2020;395(10225):689-97. DOI PubMed

23. Lessler J, Reich NG, Brookmeyer R, Perl TM, Nelson KE, Cummings DA. Incubation periods of acute respiratory viral infections: a systematic review. Lancet Infect Dis 2009;9(5):291-300. DOI PubMed

24. He $X$, Lau EH, Wu $P$, Deng $X$, Wang J, Hao X, Lau YC, Wong JY, Guan Y, Tan X, Mo X, Chen Y, Liao B, Chen W, Hu F, Zhang Q, Zhong M, Wu Y, Zhao L, Zhang F, Cowling BJ, Li F, Leung GM. Temporal dynamics in viral shedding and transmissibility of COVID-19. Nat Med 2020;26(5):672-5. DOI PubMed

25. Moghadas SM, Fitzpatrick MC, Sah P, Pandey A, Shoukat A, Singer $\mathrm{BH}$, Galvani AP. The implications of silent transmission for the control of COVID-19 outbreaks. Proc Natl Acad Sci USA 2020;117(30):17513-5. DOI PubMed 


\section{Annexes : Tableaux}

Tableau A1 : Pourcentage de positivité des cas de grippe confirmés en laboratoire à Hamilton, Ontario, au cours des semaines 1 à 26 de 2010 à 2019

\begin{tabular}{|c|c|c|c|c|c|}
\hline Semaine & Moyenne & Médiane & Quartile 1 & Quartile 3 & Écart-type \\
\hline 1 & 18,5 & 21,1 & 11,0 & 26,2 & 10,3 \\
\hline 2 & 16,0 & 15,6 & 3,6 & 29,8 & 11,2 \\
\hline 3 & 17,4 & 21,0 & 5,4 & 26,1 & 10,0 \\
\hline 4 & 16,5 & 12,2 & 5,8 & 26,6 & 11,9 \\
\hline 5 & 16,8 & 17,3 & 8,8 & 24,9 & 10,3 \\
\hline 6 & 17,5 & 16,7 & 12,7 & 23,8 & 9,2 \\
\hline 7 & 15,2 & 16,0 & 7,4 & 21,5 & 8,8 \\
\hline 8 & 15,9 & 15,2 & 6,8 & 23,8 & 9,3 \\
\hline 9 & 14,6 & 13,9 & 8,2 & 20,7 & 9,1 \\
\hline 10 & 14,8 & 14,2 & 5,8 & 20,0 & 10,1 \\
\hline 11 & 12,7 & 11,2 & 3,9 & 21,6 & 8,5 \\
\hline 12 & 10,2 & 10,9 & 5,1 & 16,0 & 6,2 \\
\hline 13 & 11,4 & 10,9 & 5,6 & 18,4 & 6,8 \\
\hline 14 & 11,5 & 9,8 & 3,6 & 20,3 & 8,4 \\
\hline 15 & 8,5 & 7,6 & 4,1 & 11,7 & 5,8 \\
\hline 16 & 7,4 & 5,4 & 2,5 & 13,1 & 5,9 \\
\hline 17 & 7,3 & 5,8 & 2,2 & 11,9 & 5,6 \\
\hline 18 & 5,5 & 6,3 & 2,2 & 7,1 & 3,4 \\
\hline 19 & 3,2 & 2,0 & 0,9 & 5,8 & 3,2 \\
\hline 20 & 3,3 & 1,6 & 0,7 & 6,3 & 3,3 \\
\hline 21 & 1,9 & 0,7 & 0,0 & 2,4 & 3,0 \\
\hline 22 & 1,4 & 0,7 & 0,0 & 3,0 & 1,5 \\
\hline 23 & 1,2 & 0,0 & 0,0 & 2,1 & 1,9 \\
\hline 24 & 0,8 & 0,0 & 0,0 & 1,9 & 1,1 \\
\hline 25 & 0,3 & 0,0 & 0,0 & 0,3 & 0,6 \\
\hline 26 & 0,0 & 0,0 & 0,0 & 0,0 & 0,0 \\
\hline
\end{tabular}

Tableau A2 : Pourcentage de positivité des cas de grippe, de virus respiratoire syncytial, de métapneumovirus, de virus para-influenza, d'adénovirus et de rhinovirus/entérovirus confirmés en laboratoire à Hamilton, Ontario, au cours des semaines 1 à 26 de 2020

\begin{tabular}{|l|r|r|r|r|r|r|} 
Semaine & $\begin{array}{c}\text { Pourcentage de } \\
\text { positivité pour } \\
\text { la grippe }\end{array}$ & $\begin{array}{c}\text { Pourcentage } \\
\text { de positivité } \\
\text { du virus } \\
\text { respiratoire } \\
\text { syncytial }\end{array}$ & $\begin{array}{c}\text { Pourcentage } \\
\text { de positivité du } \\
\text { métapneumovirus }\end{array}$ & $\begin{array}{c}\text { Pourcentage de } \\
\text { positivité pour } \\
\text { le virus para- } \\
\text { influenza }\end{array}$ & $\begin{array}{c}\text { Pourcentage } \\
\text { de positivité de } \\
\text { l'adénovirus }\end{array}$ & $\begin{array}{c}\text { Pourcentage } \\
\text { de positivité } \\
\text { du rhinovirus/ } \\
\text { entérovirus }\end{array}$ \\
\hline 1 & 17,7 & 5,9 & 0,5 & 0,0 & 0,5 & 2,2 \\
\hline 2 & 9,6 & 5,6 & 0,8 & 0,4 & 0,8 & 1,6 \\
\hline 3 & 9,6 & 1,8 & 0,9 & 0,4 & 0,4 & 3,9 \\
\hline 4 & 11,5 & 5,8 & 0,8 & 0,0 & 0,4 & 1,2 \\
\hline
\end{tabular}


Tableau A2 : Pourcentage de positivité des cas de grippe, de virus respiratoire syncytial, de métapneumovirus, de virus para-influenza, d'adénovirus et de rhinovirus/entérovirus confirmés en laboratoire à Hamilton, Ontario, au cours des semaines 1 à 26 de 2020 (suite)

\begin{tabular}{|c|c|c|c|c|c|c|}
\hline Semaine & $\begin{array}{l}\text { Pourcentage de } \\
\text { positivité pour } \\
\text { la grippe }\end{array}$ & $\begin{array}{l}\text { Pourcentage } \\
\text { de positivité } \\
\text { du virus } \\
\text { respiratoire } \\
\text { syncytial }\end{array}$ & $\begin{array}{c}\text { Pourcentage } \\
\text { de positivité du } \\
\text { métapneumovirus }\end{array}$ & $\begin{array}{l}\text { Pourcentage de } \\
\text { positivité pour } \\
\text { le virus para- } \\
\text { influenza }\end{array}$ & $\begin{array}{l}\text { Pourcentage } \\
\text { de positivité de } \\
\text { l'adénovirus }\end{array}$ & $\begin{array}{l}\text { Pourcentage } \\
\text { de positivité } \\
\text { du rhinovirus/ } \\
\text { entérovirus }\end{array}$ \\
\hline 5 & 6,2 & 4,5 & 0,7 & 0,0 & 0,3 & 2,4 \\
\hline 6 & 10,4 & 2,3 & 1,2 & 0,4 & 0,8 & 1,5 \\
\hline 7 & 5,8 & 1,9 & 1,0 & 0,0 & 1,0 & 1,4 \\
\hline 8 & 8,8 & 2,4 & 2,4 & 0,0 & 0,0 & 2,4 \\
\hline 9 & 3,2 & 0,5 & 0,5 & 0,0 & 0,5 & 0,0 \\
\hline 10 & 5,8 & 1,5 & 1,9 & 1,0 & 1,0 & 4,4 \\
\hline 11 & 5,1 & 0,6 & 2,4 & 0,4 & 0,4 & 6,1 \\
\hline 12 & 0,0 & 0,0 & 0,5 & 0,2 & 0,0 & 0,2 \\
\hline 13 & 0,1 & 0,0 & 0,1 & 0,1 & 0,0 & 0,2 \\
\hline 14 & 0,0 & 0,0 & 0,0 & 0,0 & 0,0 & 0,0 \\
\hline 15 & 0,0 & 0,0 & 0,0 & 0,0 & 0,0 & 0,0 \\
\hline 16 & 0,0 & 0,0 & 0,0 & 0,0 & 0,0 & 0,0 \\
\hline 17 & 0,0 & 0,0 & 0,0 & 0,0 & 0,0 & 0,0 \\
\hline 18 & 0,0 & 0,0 & 0,0 & 0,0 & 0,0 & 0,0 \\
\hline 19 & 0,0 & 0,0 & 0,0 & 0,0 & 0,0 & 0,0 \\
\hline 20 & 0,0 & 0,0 & 0,0 & 0,0 & 0,0 & 0,0 \\
\hline 21 & 0,0 & 0,0 & 0,0 & 0,0 & 0,0 & 0,0 \\
\hline 22 & 0,0 & 0,0 & 0,0 & 0,0 & 0,0 & 0,0 \\
\hline 23 & 0,0 & 0,0 & 0,0 & 0,0 & 0,0 & 0,0 \\
\hline 24 & 0,0 & 0,0 & 0,0 & 0,0 & 0,0 & 0,0 \\
\hline 25 & 0,0 & 0,0 & 0,0 & 0,0 & 0,0 & 0,0 \\
\hline 26 & 0,0 & 0,0 & 0,0 & 0,0 & 0,0 & 0,0 \\
\hline
\end{tabular}

Tableau A3 : Paramètres estimés du modèle à partir de l'inférence bayésienne

\begin{tabular}{|l|r|r|r|}
\multicolumn{1}{|c|}{ Paramètre } & Moyenne & $95 \%$ & Intervalle de crédibilité \\
\hline$\beta 1$ & 13,494 & $-0,891$ & 21,695 \\
\hline$\beta 2$ & 0,114 & $-0,888$ & 1,985 \\
\hline$\sigma$ & 4,005 & 1,932 & 5,102 \\
\hline
\end{tabular}

